\title{
Staining Pattern of the Brush Border and Detection of Cytoplasmic Granules in the Uriniferous Tubules of Female DBA/2Cr Mouse Kidney: Comparison among Various Fixations and Stains
}

\author{
Akira YABUKI ${ }^{1}$, Syusaku SUZUKI ${ }^{2}$, Mitsuharu MATSUMOTO ${ }^{1)}$, Masamichi KUROHMARU ${ }^{3)}$, Yoshihiro HAYASHI ${ }^{3)}$ \\ and Hayao NISHINAKAGAWA ${ }^{1)}$ \\ ${ }^{1)}$ Department of Veterinary Anatomy, Faculty of Agriculture, Kagoshima University, Kagoshima 890-0065, ${ }^{2}$ Institute of Laboratory \\ Animal Sciences, Faculty of Medicine, Kagoshima University, Kagoshima 890-0075 and ${ }^{3)}$ Department of Veterinary Anatomy, Graduate \\ School of Agricultural and Life Sciences, The University of Tokyo, Tokyo 113-8657, Japan
}

(Received 2 May 2001/Accepted 15 August 2001)

ABSTRACT. The proximal straight tubules of the female mouse kidney exhibit heavy periodic acid Schiff (PAS) staining in their brush borders and numerous cytoplasmic granules. In the present study, the female DBA/2Cr mouse kidney was examined, using various fixatives (formalin, PFA, PLP, Zamboni's, Bouin, or Carnoy solution) and various staining methods (HE, PAS, alcian blue, periodic acid methenamine-silver (PAM), toluidine blue, azan, or Congo red). Under azan and PAM, the staining pattern of the brush border was similar to that of PAS, and few effects of the fixative were observed. Cytoplasmic granules were clearly detected with PAM as well as PAS. However, these granules were not detected with Carnoy solution. Furthermore, distribution of granules differed between PAS and PAM. KEY WORDS: female DBA/2Cr, fixatives and stain, kidney.

J. Vet. Med. Sci. 63(12): 1339-1342, 2001

We recently reported sexual dimorphisms in the proximal straight tubules (PST) of ICR mice [8]. The brush border of the PST was stained more intensely with PAS in females than in males, and PAS-positive granules were observed in the female PST epithelium. Sexual dimorphisms in the PST similar to those in ICR mice were also identified in BALB/ cA, C57BL/6J, C3H/HeN, and DBA/2Cr mouse strains [9]. Among these mice, female DBA/2Cr showed particularly remarkable features. Briefly, giant granules greater than nuclear size were frequently observed in addition to many small granules [7, 9]. However, investigation of these structural features using various fixatives has not previously been attempted, despite the known variations in histological features of mammalian tissues under different fixatives $[1,5$, 6]. Likewise, observation using various stains has not previously been reported. Therefore, it is unclear whether heavy staining in the brush border and cytoplasmic granules of the PST of female mouse kidney can be detected under different fixative and stain combinations, and whether the distribution of cytoplasmic granules varies under these conditions. To address this issue, we performed histological observations of female DBA/2Cr mouse kidney using six fixatives and seven stains.

The present study was carried out in accordance with the Guidelines for Animal Experimentation of the Faculties of Medicine and Agriculture, Kagoshima University. Six female DBA/2Cr Slc mice were housed in an open system room with a one-way airflow system (temperature, $22 \pm$ $1^{\circ} \mathrm{C}$; humidity, $55 \pm 10 \%$; light period, 07:00 hr-19:00 hr; ventilation, 12 times/hr) in the Institute of Laboratory Animal Sciences, Faculty of Medicine, Kagoshima University. The mice received an autoclaved commercial diet (CE-2, Japan CLEA, Japan) and tap water ad libitum. Three mice were dissected by exsanguination under anesthesia (a mixture of ketamine and medetomidine). Kidneys were quickly removed, sliced, and then immersed in $10 \%$ neutral formalin in $0.1 \mathrm{M}$ phosphate buffer (PB) (formalin), $4 \%$ paraformaldehyde in PB (PFA), periodate-lysine-paraformaldehyde (PLP), Zamboni's, Bouin's or Carnoy solution. Fixations were performed at room temperature for $2 \mathrm{hr}$ in Carnoy solution or for 2 days in other fixatives. Under anesthesia, the remaining three mice were perfused with cold physiological saline for $30 \mathrm{sec}$ and then with cold formalin for 10 min. The kidneys were then removed and sliced. The slices were again immersed in the same fixative for 2 days at $4{ }^{\circ} \mathrm{C}$. After fixation, the kidney slices were routinely embedded in paraffin, cut into $3-\mu$ m-thick sections, stained with hematoxylin-eosin (HE), PAS, alcian blue, PAM, toluidine blue, azan, or Congo red, and observed by light microscopy. Staining intensity of the brush borders of the proximal convoluted tubules (PCT) or the PST was semiquantitatively examined. Briefly, staining intensity was graded from 0 to $5(0=$ not stained; $1=$ very weak staining; $2=$ =weak staining; 3 =moderate staining; 4=strong staining; 5=heavy staining), and mean staining intensities were then classified as follows: -, mean $\leq 1.0 ;+, 1.0<$ mean $\leq 2.0 ;++, 2.0<$ mean $\leq 3.0$; ,$+++ 3.0<$ mean $\leq 4.0 ;++++$, mean $>4.0$. The number of small cytoplasmic granules in the PCT or PST was calculated according to a previously reported method [9]. Values were expressed as the mean \pm standard error (S.E.), and statistical significance was defined as $\mathrm{p}<0.01$, using one-way analysis of variance (ANOVA, Bonferron/Dunn test).

Staining intensity in the brush borders of the PCT or PST is shown in Table 1. Under PAS staining, the brush border of the PST was stained more intensely than that of the PCT, regardless of fixation method (Fig. 1a). Similar segmental 
Table 1. Staining intensity in the brush border of the proximal tubules

\begin{tabular}{|c|c|c|c|c|c|c|c|c|c|c|c|c|c|c|}
\hline & \multicolumn{2}{|c|}{ Formalin* } & \multicolumn{2}{|c|}{ Formalin } & \multicolumn{2}{|c|}{ PFA } & \multicolumn{2}{|c|}{ PLP } & \multicolumn{2}{|c|}{ Zamboni's } & \multicolumn{2}{|c|}{ Bouin's } & \multicolumn{2}{|c|}{ Carnoy } \\
\hline & PCT & PST & PCT & PST & PCT & PST & PCT & PST & PCT & PST & PCT & PST & PCT & PST \\
\hline $\mathrm{HE}$ & ++ & ++ & ++ & ++ & ++ & ++ & ++ & ++ & ++ & ++ & ++ & ++ & ++ & ++ \\
\hline PAS & ++ & ++++ & + & ++++ & ++ & ++++ & + & ++++ & + & ++++ & + & ++++ & + & ++++ \\
\hline Alcian blue & - & - & - & - & - & - & - & - & - & - & - & - & - & - \\
\hline PAM & ++ & +++ & ++ & ++++ & ++ & +++ & ++ & +++ & ++ & +++ & ++ & ++++ & + & ++++ \\
\hline Toluidine blue & + & + & + & + & + & + & + & + & - & - & - & - & - & - \\
\hline Azan & + & +++ & + & +++ & + & ++ & + & ++ & + & +++ & + & +++ & + & +++ \\
\hline Congo red & - & - & - & - & - & - & - & - & - & - & - & - & - & - \\
\hline
\end{tabular}

*: perfusion. PCT: proximal convoluted tubules. PST: proximal straight tubules.
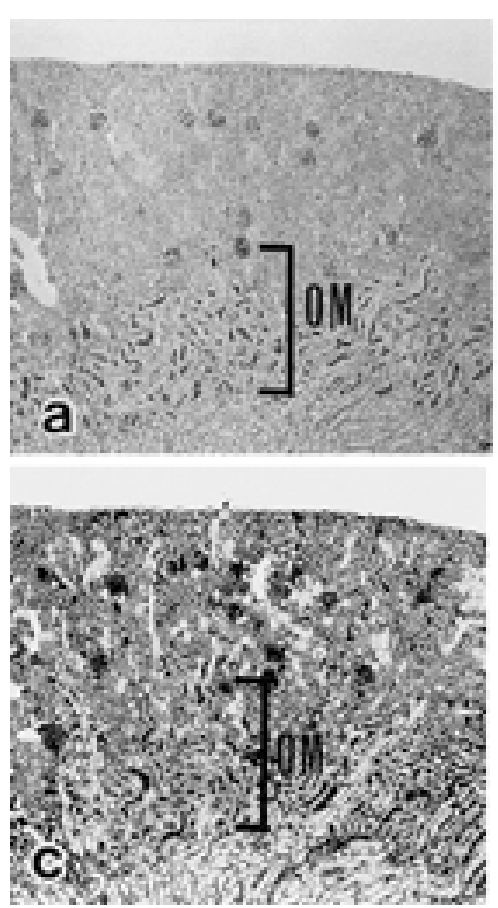

differences were observed upon staining with PAM or azan (positive for anilin blue). Again, the brush border staining in the PST was more intense than in the PCT (Fig. 1b and c). No segmental differences were observed in the brush border after staining with $\mathrm{HE}$, alcian blue, toluidine blue, or Congo red. Slight effects of fixatives on staining intensity of the brush border were observed in both PCT and PST. With formalin fixation, perfusion and immersion techniques produced similar staining patterns and segmental differences.

Table 2 shows the number of small cytoplasmic granules in each segment of the proximal tubules. PAS staining in combination with all fixatives except for Carnoy solution revealed numerous small granules in the PST (Fig. 2). PAS staining in combination with Bouin's solution revealed fewer granules than PAS staining in combination with formalin, PFA or PLP. In addition, the number of granules revealed by PAS staining in combination with PLP or Zamboni's solution was significantly lower than that revealed by PAS staining in combination with formalin. In the PCT, few positive granules were revealed by PAS staining in combi-

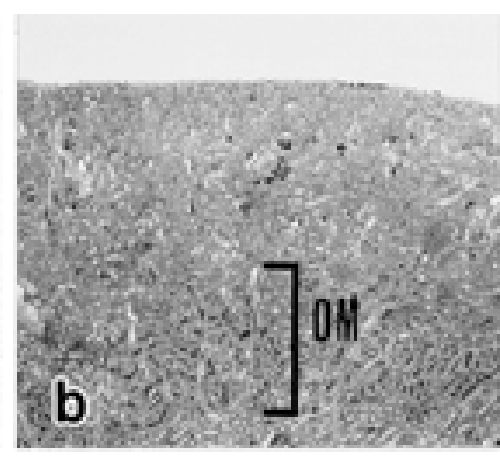

Fig. 1. Light micrographs of a kidney upon Carnoy fixation. a: PAS stain. b: azan stain. c: PAM stain. The brush borders of the proximal straight tubules, which run throughout the outer medulla $(\mathrm{OM})$, show heavy staining.

nation with formalin, PFA or PLP. No PAS-positive granules were observed in any segments after Carnoy fixation (Fig. 2d). PAM staining in combination with all fixatives except for Carnoy solution also revealed numerous small granules in the PCT and PST (Fig. 3), and the number of granules was significantly greater than that revealed by PAS staining. Furthermore, PAM-positive granules were also detected in the thin limbs of the loop of Henle, thick ascending limbs, distal convoluted tubules, and collecting ducts (Fig. 3). No PAM-positive granules were detected in any of uriniferous tubules after Carnoy fixation. Small cytoplasmic granules were detected only in response to PAS and PAM staining. Prevalence and distribution of PAS- or PAM-positive small granules were not affected by formalin fixation method (immersion vs. perfusion).

The staining pattern of giant cytoplasmic granules, localized in the PST, is shown in Table 2. Intense staining of giant granules was observed in response to PAS or PAM except after fixation with Carnoy solution (Figs. 2 and 3). Weak staining of giant granules was observed in response to 
Table 2. Number of small granules in the proximal tubules $\left(10,000 \mu \mathrm{m}^{2}\right)$

\begin{tabular}{|c|c|c|c|c|c|c|c|c|c|c|c|c|c|c|}
\hline & \multicolumn{2}{|c|}{ Formalin* } & \multicolumn{2}{|c|}{ Formalin } & \multicolumn{2}{|c|}{ PFA } & \multicolumn{2}{|c|}{ PLP } & \multicolumn{2}{|c|}{ Zamboni's } & \multicolumn{2}{|c|}{ Bouin's } & \multicolumn{2}{|c|}{ Carnoy } \\
\hline & PCT & PST & PCT & PST & PCT & PST & PCT & PST & PCT & PST & PCT & PST & PCT & PST \\
\hline $\mathrm{HE}$ & - & - & - & - & - & - & - & - & - & - & - & - & - & - \\
\hline PAS & $\begin{array}{c}56.3 \\
(13.8)\end{array}$ & $\begin{array}{c}657.6^{\mathrm{f}} \\
(56.7)\end{array}$ & $\begin{array}{c}85.4 \\
(10.1)\end{array}$ & $\begin{array}{l}867.8^{\text {det }} \\
(23.5)\end{array}$ & $\begin{array}{c}48.8 \\
(14.3)\end{array}$ & $\begin{array}{r}659.7^{\mathrm{f}} \\
(65.4)\end{array}$ & $\begin{array}{c}54.0 \\
(12.8)\end{array}$ & $\begin{array}{c}576.5 b^{f} \\
(11.2)\end{array}$ & - & $\begin{array}{c}464.6^{b f} \\
(26.7)\end{array}$ & - & $\begin{array}{l}259.5^{\text {abcd }} \\
(29.0)\end{array}$ & - & - \\
\hline Alcian blue & - & - & - & - & - & - & - & - & - & - & - & - & - & - \\
\hline PAM & $\begin{array}{c}543.4 \\
(106.3)\end{array}$ & $\begin{array}{r}1327.0 \\
(82.8)\end{array}$ & $\begin{array}{l}704.5 \\
(71.1)\end{array}$ & $\begin{array}{l}1283.1 \\
(160.7)\end{array}$ & $\begin{array}{l}755.4 \\
(69.7)\end{array}$ & $\begin{array}{r}1473.7 \\
(94.5)\end{array}$ & $\begin{array}{l}603.5 \\
(65.7)\end{array}$ & $\begin{array}{l}1523.4 \\
(119.3)\end{array}$ & $\begin{array}{l}622.1 \\
(43.4)\end{array}$ & $\begin{array}{r}1432.6 \\
(45.7)\end{array}$ & $\begin{array}{l}625.2 \\
(83.6)\end{array}$ & $\begin{array}{l}1079.3 \\
(125.5)\end{array}$ & - & - \\
\hline Toluidine blue & - & - & - & - & - & - & - & - & - & - & - & - & - & - \\
\hline Azan & - & - & - & - & - & - & - & - & - & - & - & - & - & - \\
\hline Congo red & - & - & - & - & - & - & - & - & - & - & - & - & - & - \\
\hline
\end{tabular}

*: perfusion. PCT: proximal convoluted tubules. PST: proximal straight tubules. Values expressed as mean (S.E.). -: not detected. ${ }^{\text {a-f }}$ indicates significant differences between fixatives $(\mathrm{p}<0.01):{ }^{\mathrm{a}} \mathrm{vs}$ formalin (perfusion), ${ }^{\mathrm{b}}$ vs formalin, ${ }^{\mathrm{c}} \mathrm{vs}$ PFA, ${ }^{\mathrm{d}} \mathrm{vs}$ PLP, ${ }^{\mathrm{e}} \mathrm{vs}$ Zamboni's, ${ }^{\mathrm{f}} \mathrm{vs}$ Bouin's.

Table 3. Staining pattern of giant granules in the proximal straight tubules

\begin{tabular}{lccccccc}
\hline & Formalin* & Formalin & PFA & PLP & Zamboni's & Bouin's & Carnoy \\
\hline HE & very weak & very weak & very weak & very weak & very weak & very weak & NS \\
PAS & strong & strong & strong & strong & strong & strong & NS \\
Alcian blue & NS & NS & NS & NS & NS & NS & NS \\
PAM & strong & strong & strong & strong & strong & strong & NS \\
Toluidine blue & weak & weak & weak & weak & weak & weak & NS \\
Azan & very weak & very weak & very weak & very weak & very weak & weak & NS \\
Congo red & NS & NS & NS & NS & NS & NS & NS \\
\hline *. & & & & & &
\end{tabular}

*: perfusion. NS: not stained.

HE (eosinophilic), azan (positive for anilin blue), or toluidine blue. However, no staining was observed in response to alcian blue or Congo red. No staining differences were observed between immersion- and perfusion-fixation using formalin.

Histological features of the PST epithelium of the female mouse kidney were recently clarified [7-9]. In the present study, we examined the kidneys of female DBA/2Cr mice, using six fixatives and seven stains. In the PAS, PAM and azan stains, staining intensity of the brush border of the PST was stronger than that of the PCT, and PAS and PAM stains also revealed cytoplasmic granules in the PST. Although segmental differences of brush border staining were similar among the fixatives, detection of the granules was clearly affected by fixative type. Therefore, we concluded that the strength of staining in the brush border of PST is not related to the presence of cytoplasmic granules but rather to the amount or chemical characteristics of the glycocalyx that coats the microvilli.

Detection of PAS-positive small and giant granules was clearly affected by the fixative type. The results indicate that Carnoy and Bouin's solutions are not suitable for detection of PAS-positive granules, especially Carnoy solution. Carnoy solution is a mixture of alcohol, chloroform and acetic acid, and Bouin's solution is a mixture of formaldehyde, picric acid and acetic acid. When alcohol was used as the dehydration reagent, aldehyde fixatives showed good detection of the granules, and the number of granules detected after fixation with Zamboni's solution (aldehyde fixative
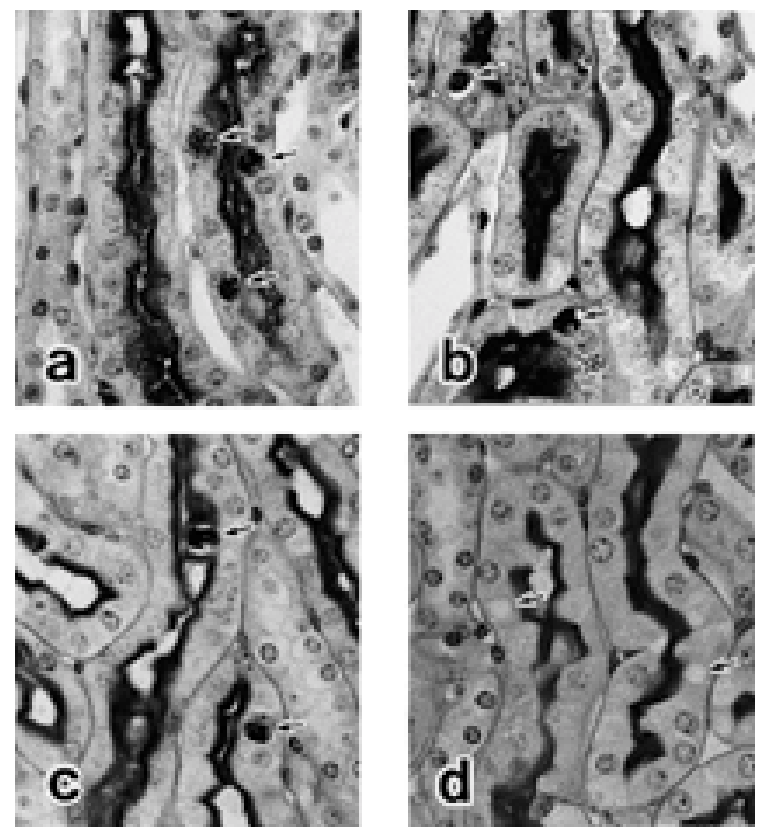

Fig. 2. Light micrographs of proximal straight tubules stained with PAS. a: formalin fixation (perfusion). b: formalin fixation (immersion). c: Bouin's fixation. d: Carnoy fixation. Arrows indicate the giant granules. Fewer small granules are observed after Bouin' fixation (c) than after formalin fixation (a and b). Small and giant granules are not detected after Carnoy fixation (d). 


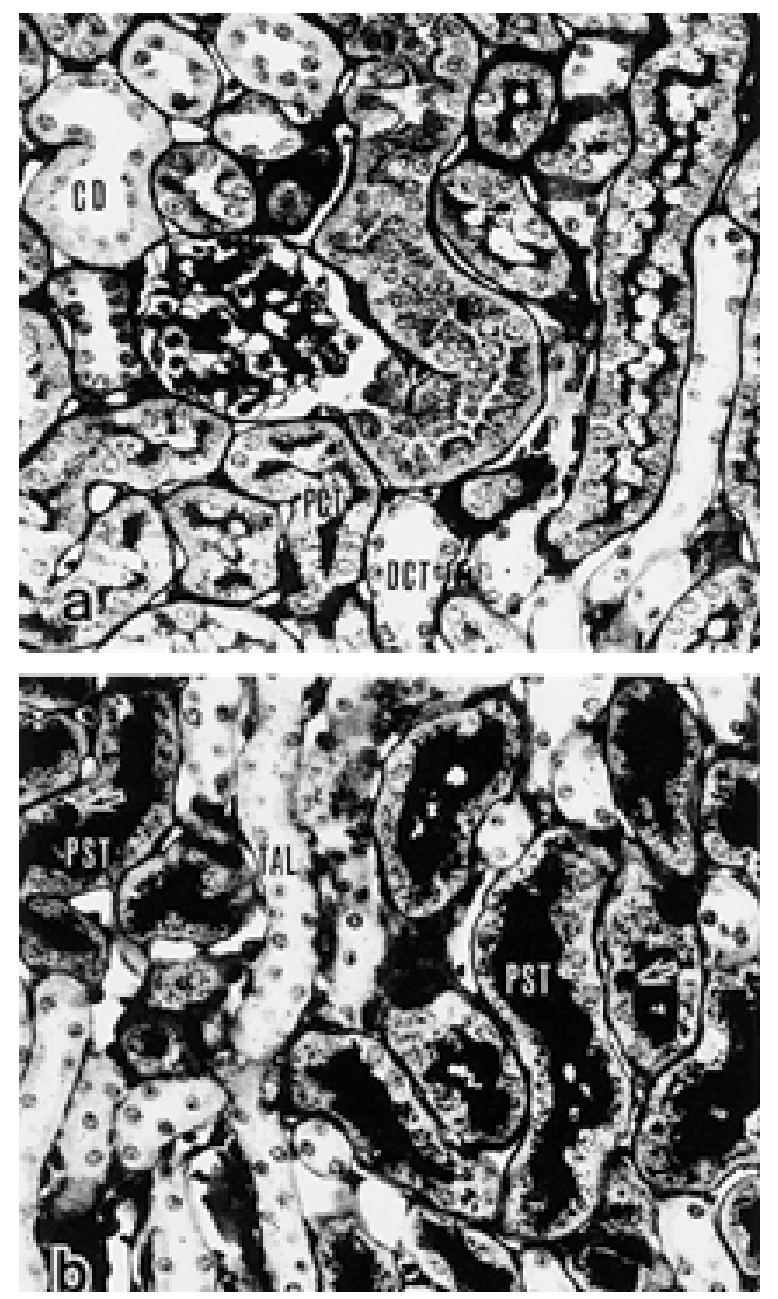

Fig. 3. Light micrographs of a kidney stained with PAM. Perfusion-formalin fixation. a: cortex. b: outer medulla. PAM-positive cytoplasmic granules are very numerous in the proximal straight tubules (PST) and moderately numerous in proximal convoluted tubules (PCT). A few PAM-positive granules are detected in the thick ascending limb (TAL), distal convoluted tubule (DCT) and collecting duct (CD). Giant granules (arrows) in the PST are PAM-positive.

containing picric acid) was significantly less than the number detected after fixation with formalin. This suggests that PAS staining of the substances contained in the granules was inhibited by chloroform, picric acid and/or acetic acid. However, the chemical composition of the substances responsible for this is unknown at present.

We observed similar staining patterns in PAS- and PAMstained samples. Because PAM and PAS staining identified analogous small and giant granules, we initially presumed that the granules were identical. However, the distribution of these granules was clearly different. PAS-positive granules were highly localized in the PST, whereas PAM-positive granules showed a wide distribution. Our previous electron microscopic investigations demonstrated that PASpositive small and giant granules were electron-dense multilamellar bodies showing acid phosphatase activity, and we presumed these granules were lysosomes $[8,9]$. Furthermore, previous studies demonstrated that several lysosomal enzymes, (e.g., acid phosphatase and dipeptidyl peptidase) contain high numbers of carbohydrate moieties [2-4]. Given this evidence, we propose that PAS and PAM stain identical substances contained in lysosomes, but that sensitivity of PAM staining is higher than that of PAS staining.

In summary, we examined female DBA/2Cr mouse kidneys using six fixatives and seven stains, and we observed that the type of fixative or stain affected the staining pattern of the brush border and detection of cytoplasmic granules. We believe that the present findings have significant implications for histological observation of kidneys of female mice.

\section{REFERENCES}

1. Dutt, M.K. 1979. Microsc. Acta 81: 379-382.

2. Kyouden, T., Himeno, M., Ishikawa, T., Ohsumi, Y. and Kato, Y. J. 1992. J. Biochem (Tokyo) 111: 770-777.

3. Himeno, M., Koutoku, H., Ishikawa, T. and Kato, K. 1989. J. Biochem (Tokyo) 105: 449-456.

4. Himeno, M., Koutoku, H., Tsuji, H. and Kato, K. 1988. J. Biochem (Tokyo) 104: 773-776.

5. Ruitenberg, E.J., Gustowska, L., Elgersma, A. and Ruitenberg, H.M. 1982. Int. Arch. Allergy. Appl. Immunol. 67: 233-238.

6. Stickland, N.C. 1975. Stain. Technol. 50: 255-264.

7. Yabuki, A., Maeda, M., Suzuki, S., Matsumoto, M., Kurohmaru, M., Hayashi, Y., Taniguchi, K. and Nishinakagawa, H. 2001. J. Vet. Med. Sci. 63: 461-465.

8. Yabuki, A., Suzuki, S., Matsumoto, M. and Nishinakagawa, H. 1999. Anat. Rec. 255: 316-323.

9. Yabuki, A., Suzuki, S., Matsumoto, M. and Nishinakagawa, H. 2001. Exp. Anim (Tokyo) 50: 59-66. 